\title{
The Modified Model of Q-learning Search Strategy Based on LDA-DBN
}

\author{
Shihao Zhu ${ }^{1}$, Guangfeng Zhang ${ }^{2, *}$ and Dongfan $\mathrm{Zhao}^{3}$ \\ ${ }^{1}$ School of mathematics, Jilin University, Changchun, Jilin, China \\ ${ }^{2}{ }^{3}$ College of computer science and technology, JilinUniversity, Changchun, Jilin, China \\ * Corresponding Author
}

\begin{abstract}
The network in web has the characteristic of information roughness. Focused crawling is able to grab the information selectively when topic relate to web page. In this paper, utilizing the characteristics of Gibbs using in DBN and LDA, in the sliding window containing the URL link to semantic recognition, the prediction of $Q$ - learning can be used in LDADBN hybrid model, that is, when the URL link in the web pages.
\end{abstract}

\section{Keywords-focused crawling; q-learning; LDA; DBN}

\section{INTRODUCTION}

A With the popularity of the network and the significant growth of social media, the scale of web information are rapid expanding [1]. Naturally, the demand for customer experience increase prominently when uses the search engine, such as professionalism, accuracy, efficiency, timeliness. Focused crawler is proposed to solve the problem. Compared with the common crawlers, topic crawler can select filter-topic links and choose suitable page orientation or related themes to mining. Effectively, searching strategies of topic crawler can consider as a decision problem of search the essay's semantic information in rough attributes graph. Thus, the retrieval precision is concerned usually.

Inspired by the dirichlet distribution model, we not only considers the document on the spatial dimension in a dictionary, but also take subject dimension into consideration, associated texts through the implied theme [2]. The model reduces the dimension of the document, thence, the implicit semantics hidden behind the text can be delved. We also aroused by deep belief network model, it directly overcomes the inefficient problem caused by the superposition of multilayer network. Therefore, it make things easier to learn hidden knowledge. In terms of processing information, compared to other methods, artificial intelligence is more similar to the process of the human brain.

Combined with emerging technologies above, this paper proposes a model of theme crawler search strategy. The main idea is the predicting part of the Q-learning is adopt the LDA DBN hybrid model. Schematic, when score for URL links, contained within the sliding window, use semantics of the web information to identify, so that improve intelligent of the topic crawler.

\section{RELATED WORK}

At present, most of the crawler algorithm is based on the optimal priority crawler, the difference among them is only the heuristic rules of ranking non-visited URL. In addition to assigned a different priority to non-access points, we can also change the priority strategies to improve the performance of simple best-first reptiles. Such as accessing some lower value, it may lead to a high quality web page. In detail, it tends to have better results than strict adherence crawling optimal policy priority order of reptiles, a phenomenon reflected in the optimal $\mathrm{N}$ priority crawlers. Intelligent crawler uses statistical models to learn and to assign weight to URL in queue. Recently, a crawler using bayesian interest factor works well through deriving a number of different characteristics to accumulate, storing the new evidence and recalculating each interest factor subsequently. Through such process, intelligent crawler can adjust themselves adapting to web content and link structure which have visited. It also can be a real-time training apprentice in running, named reinforcement learning, which has been adopted by several crawler combined with different characteristics and different learning methods. In reinforcement learning, there is a network in which the node is the state and directional link is all sorts of behavior [3]. It need the mapping from state of learning to the behavior and makes the future reward is the largest.

\section{PROBLEM ANALYSIS MODELING}

\section{A. Work Frame of the Topic Crawler}

Assumes that the web page contains a reliable clue which characterization other web content. This is a necessary condition to design the crawler, which is reason better than random selection, to crawl the specific content. In fact, if it can't make an assessment to non-visited web page, then crawler only walks on the web graph randomly or full traversal. Practically, crawl algorithm takes advantage of the clues and the hyperlinks hidden in the words, by using lexical analysis and link topology analysis respectively. The former determines whether two pages on the words with similar characteristics. The latter determines the length of the link between distances.

To reduce the complexity of problem, the web network can be formalized as following description, the Internet web can be abstracted as a rough attribute graph model, the object and attributes are described as nodes and the relationship between 
the object represent with lines. It describes incomplete information and dynamic link within complex network. When the topic crawler walks on the rough attributes graph, it can be seen as an autonomous agent. What the Q-learning can solve is how to learning to choose optimal action achieving the goal when an autonomous agent is able to perceive the environment. When topic crawler makes every actions in their environment, the environment will provide a reward or punishment information to show the result status correct or not. It is noteworthy that the task of topic crawler is to learn from the indirect, delayed return environment, so as to produce the largest cumulative returns in the subsequent action. In the Qlearning, above-mentioned, we can make use of DLA - DBN optimizing learning estimate when the topic crawler walk on the rough attribute graph. Essentially, learning model by use LDA model is able to identify the semantic text node. Corresponding, utilizing DBN enhances semantic recognition effect. The full process is shown in Figure I.

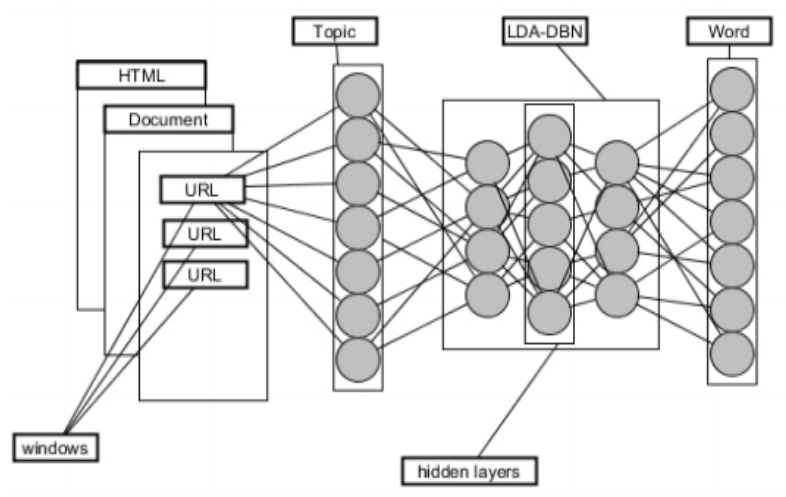

FIGURE I. TOPIC CRAWLER PROCESS DIAGRAM

When topic dictionary is large enough, meeting the needs of user's input keywords, a dictionary include most of the keywords about a subject. Effectively, in this model is to address the issue of an essay of this retrieval recognition.

\section{B. Using Q - Learning to Search}

In the process of the crawler travel, different states of the set $\mathrm{S}$ perceived environment and set $\mathrm{A}$ represent executable actions. At each discrete time step $\mathrm{t}$, crawler perceives the current state of $s_{t}$, selects the current action $a_{t}$ to execute. When environment response the crawler, it rewards $r_{t}=r\left(s_{t}, a_{t}\right)$, and generates a successor state $S_{t}+1=\delta\left(s_{t}, a_{t}\right)$. Evaluation function $\mathrm{Q}(\mathrm{s}, \mathrm{a})$ is defined as: its value is from the state $\mathrm{s}$ and a maximum conversion using the action as the first action when cumulative returns. The learning task of crawler is to study an optimal strategy of $\pi^{*}$, makes for all state $\mathrm{s}$, accumulating return $\mathrm{V}^{*}\left(\mathrm{~s}_{\mathrm{t}}\right)$ to maximum:

$$
\pi^{*} \equiv \arg \max _{\pi} V^{*}(s),(\forall s)
$$

$$
V^{*}\left(s_{t}\right) \equiv r_{t}+\gamma r_{t+1}+\gamma^{2} r_{t+2}+\cdots=\sum_{i=0}^{\infty} \gamma^{i} r_{t+i}
$$

$\rho_{\tau+1}$ returned sequence which are generated by the start state of $\sigma_{\tau}$ and reuse the strategy to choose action, $0 \leq \gamma<1$ is a constant, determines the relative value of delay return immediately and return. Crawler only needs to consider the current state $\mathrm{s}$ at each available action $\mathrm{a}$, and choose which the $\mathrm{Q}(\mathrm{s}, \mathrm{a})$ maximize the action. This can be accomplished through an iterative approximation method. [4].

$$
V^{*}(s)=\max _{a^{\prime}} Q\left(s, a^{\prime}\right)
$$

$$
Q(s, a) \equiv r(s, a)+\gamma \max _{a^{\prime}} Q\left(\delta(s, a), a^{\prime}\right) t
$$

This recursive definition of $\mathrm{Q}$ function provides a loop approximation algorithm on the basis of Q.

\section{Using LDA Model to Judge}

After crawling related links to the pages, analysis them in semantics. Through a brief description of the document, it can retain the nature of statistical information and handle large document collection efficiently. In LDA model, the two most important parameters are the probability of each word entry under the theme relating to the distribution and the probability distribution of each text. LDA is a model for solving the optimization problem in nature, it is difficult to obtain the optimal solution. We can use Gibbs sampling method to solve the model, the model is derived to more simple. Based on this, LDA parameter estimation use MCMC method of Gibbs sampling algorithm, can be regarded as the text of the build process the reverse process [5]. That is, in a known set of text, the value of the parameter obtained by the parameter estimation.

As shown in Figure II, the crawler analysis model is the directed probability graph model. It is be determined by the parameters $(\alpha, \beta)$, in which $\alpha$ reflects the relative strength of theme implied in document collections, $\beta$ depict all probability distribution of the implied theme itself. Among $\theta \mathrm{k}$ means the probability distribution of document theme, $\phi \mathrm{k}$ means the probability distribution of key under a given theme, $\mathrm{M}$ means the number in text of the document, $\mathrm{K}$ represents the number in theme of the document collection, and $\mathrm{N}$ means the number in key words of each document [6].

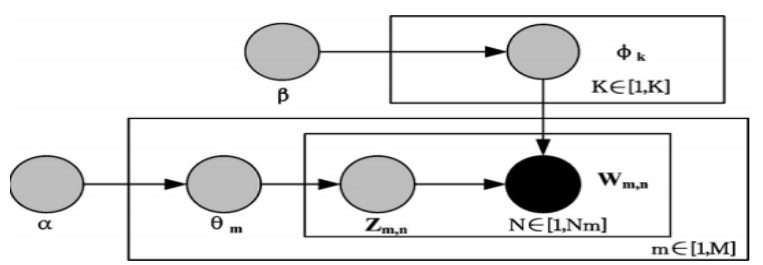

FIGURE II. THE LDA MODEL DIRECTED PROBABILITY GRAPH 
LDA probability model mainly has two main physical processes:

- $\alpha \rightarrow \theta_{\mathrm{m}} \rightarrow \mathrm{z}_{\mathrm{m}, \mathrm{n}}$ : In the generated the $\mathrm{m}_{\mathrm{th}}$ document, randomly generated a relevant document first topic parameters $\theta_{\mathrm{m}}$, then through the parameters generated document number corresponds to the $\mathrm{n}_{\text {th }}$ term in topic $\mathrm{Z}_{\mathrm{m}, \mathrm{n}}$.

- $\quad \beta \rightarrow \varphi \rightarrow\left(\omega_{\mathrm{m}, \mathrm{n}} \mid \mathrm{k}=\mathrm{Z}_{\mathrm{m}, \mathrm{n}}\right):$ Randomly generate a relevant subject and the parameters of the vocabulary $\varphi$, in the resulting parameter $\varphi$, choose topic $K=z_{m, n}$ vocabulary generated the $\mathrm{n}_{\mathrm{th}}$ word in the $\mathrm{m}_{\mathrm{th}}$ document.

According to the graph model, we can get a piece of text probability value

$$
\begin{aligned}
& p(\omega \mid \alpha, \beta)= \\
& \int p(\theta \mid \alpha)\left(\prod_{n=1}^{N} \sum_{z .} p\left(z_{n} \mid \theta\right) p\left(\omega_{n} \mid z_{n}, \beta\right)\right) d \theta
\end{aligned}
$$

By using integrating, it can avoid actual parameters, estimated in favor of sampling for each word theme. Once finalized the theme of each word, parameters can be calculated after the statistical frequency. Thus, parameter estimation problem becomes conditional probability calculated under the theme word sequence, the formula is as follows:

$$
\begin{aligned}
& p\left(\mathrm{z}_{i}={\left.\stackrel{r}{k} \mathrm{Z}_{-i}, \stackrel{r}{\omega}\right)}^{\stackrel{r}{r})}\right. \\
& =\frac{p(\stackrel{\omega}{\omega})^{r}}{p\left(\stackrel{\omega}{\omega} \mathrm{z}_{-i}\right)} \mu \frac{n_{k,-i}^{i}+\beta_{t}}{\sum_{t=1}^{V} n_{k,-i}^{i}+\beta_{t}}\left(n_{m,-i}^{t}+\alpha_{k}\right)
\end{aligned}
$$

$\mathrm{Z}_{\mathrm{i}}$ represents the theme variable of the $\mathrm{i}_{\text {th }}$ word, $\mathrm{i}$ represents not including the $\mathrm{i}_{\mathrm{th}}$ item; $\mathrm{n}_{\mathrm{k}}^{\mathrm{t}}$ says the number of term $\mathrm{t}$ in theme $\mathrm{K}, \beta_{\mathrm{t}}$ says dirichlet prior of term $\mathrm{t} ; \mathrm{n}_{\mathrm{m}}^{\mathrm{k}}$ says the number of theme $\mathrm{K}$ in text $\mathrm{m} ; \alpha_{\mathrm{k}}$ is theme dirichlet prior of theme $\mathrm{K}$. Once getting the theme label of each word, parameter calculation formula can be obtained by the following formula:

$$
f_{k, t}=\frac{n_{k}^{t}+\beta_{t}}{\sum_{t-1}^{V} n_{k}^{t}+\beta_{t}} \quad \theta_{m, k}=\frac{n_{m}^{k}+\alpha_{t}}{\sum_{k-1}^{V} n_{m}^{k}+\alpha_{t}}
$$

$\Phi_{\mathrm{k}, \mathrm{t}}$ says the probability of term $\mathrm{t}$ in theme k. $\theta_{\mathrm{m}, \mathrm{k}}$ says the probability of text $\mathrm{m}$ in theme $\mathrm{k}$. Finally obtaining the LDA model adopted by the Gibbs sampling formula:

$$
p\left(Z_{i}=k \mid \stackrel{r}{\left.Z_{-i}, \stackrel{r}{\omega}\right)}=f_{k, t} \times q_{m, k}\right.
$$

Type of $\Phi_{\mathrm{k}, \mathrm{t}}$ and $\theta_{\mathrm{m}, \mathrm{k}}$ are independent of each other, therefore, we can use DBN to training $\Phi_{\mathrm{k}, \mathrm{t}}$. The first half is still using the calculation method in LDA to calculate [7].

\section{Using DBN to Optimize}

Compared with the traditional discriminated model of neural network, based on joint distribution of the observation data and the label, evaluating P (observation | label) and P(label | observation), and discriminated model evaluate the latter only, that is, $\mathrm{P}$ (label | observation) [8].

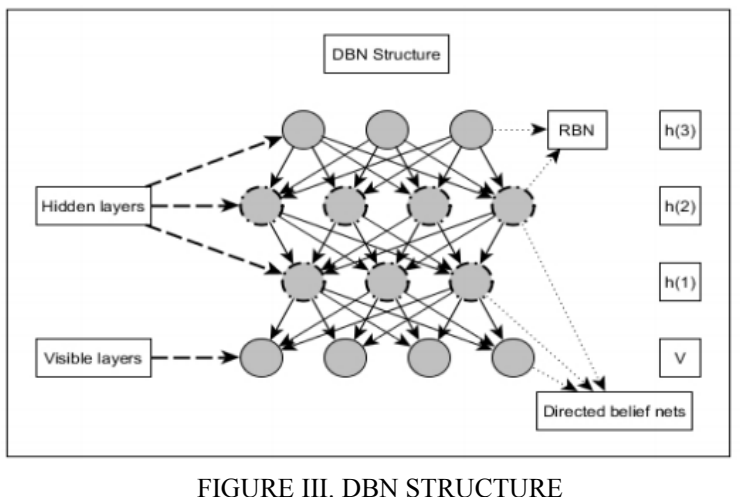

DBN is composed of multilayer RBM, the previous layer is used to constitute the associative memory. To obtain the weight of generating model by preliminary training with unsupervised stepwise greed method, called contrast differences.

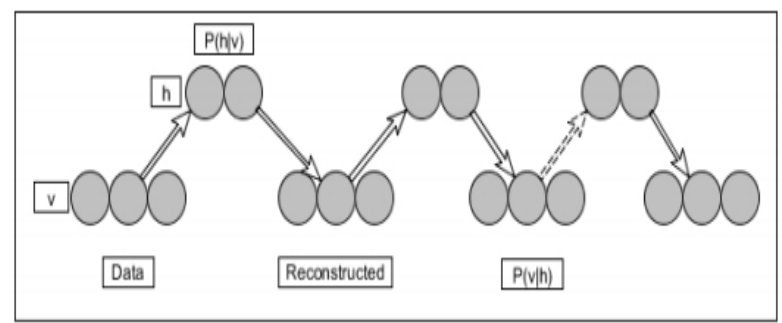

FIGURE IV. DBN GIBBS SAMPLING PROCESS DIAGRAM

During training, visual layers maps vector to the hidden units, then visual element from the hidden layer unit reconstruct. These new visual elements map again to the hidden units, which acquire a new hidden unit that Gibbs sampling. Updating the weight is based on the discrepancy between the hide elements and visual elements, in which single-step can be close to the maximum likelihood learning. Thus, it will have a significant reduction in training time. Moreover, each added layer of RBN network will improve the probability of the number of training data. After the pre-training, DBN uses a tagged data to make adjustments to determine the performance of the algorithm with BP. A set of tags will be attached to the top, through a bottom-up, to learn how to recognize the right to obtain classified face value of a network. DBNs BP algorithm only needs the right local search parameter space, the feed networks, convergence and less time compared to before. 


\section{EXPERIMENTS AND ANALYSIS}

Algorithm is implemented using MATLAB language all in this experiment, we choose the five topics from Baidu to verify, each theme crawl under the 1000 web pages, corresponding to each topic to select 50 seeds in pages. Proposed by Srinivasan harvest rate and recall rate is used to performance evaluation strategy, this strategy with the Best - First algorithm and SharkSearch algorithm is used in the comparison. Harvest rate used to estimate the precision of theme crawl Rate, harvest rate calculated as shown in formula (9), recall rate target is used to estimate the proportion of related web pages accessed over the internet, is a simulation of crawl recall and can state the performance of topic crawler algorithm. Its calculation as shown in formula (9).

$$
\text { harvestRate }=\frac{\text { relevantPages }}{\text { pagesDownload }}
$$

$$
R(t)=\frac{|C(t) \cap T|}{|T|}
$$

In the formula (9), relevant pages are numbers in the download page which are associated with the theme. Pages downloaded said crawl total pages. In formula (10), $\mathrm{R}$ represents assemble of all topics in the relevant network, $\mathrm{C}(\mathrm{t})$ is assemble obtained by web pages, $T$ is a random subset of $R$. The Best-First algorithm as the benchmark topic crawler algorithm.

TABLE I. THE AVERAGE RECALL RATE

\begin{tabular}{|l|l|c|c|c|c|c|}
\hline \multirow{2}{*}{$\begin{array}{c}\text { Algorit } \\
\text { hms }\end{array}$} & \multicolumn{7}{|c|}{ Pages download } \\
\cline { 2 - 7 } & $\mathbf{5 0 0}$ & $\mathbf{6 0 0}$ & $\mathbf{7 0 0}$ & $\mathbf{8 0 0}$ & $\mathbf{9 0 0}$ & $\mathbf{1 0 0 0}$ \\
\hline $\begin{array}{l}\text { Best- } \\
\text { First }\end{array}$ & 0.04 & 0.07 & 0.08 & 0.09 & 0.11 & 0.13 \\
\hline $\begin{array}{l}\text { Shark- } \\
\text { Search }\end{array}$ & 0.06 & 0.07 & 0.07 & 0.08 & 0.09 & 0.11 \\
\hline $\begin{array}{l}\text { LDA- } \\
\text { DBN }\end{array}$ & 0.06 & 0.07 & 0.09 & 0.11 & 0.12 & 0.14 \\
\hline
\end{tabular}

TABLEII. THE AVERAGE HARVEST RATE

\begin{tabular}{|l|c|c|c|c|c|c|}
\hline \multirow{2}{*}{$\begin{array}{c}\text { Algorit } \\
\text { hms }\end{array}$} & \multicolumn{7}{|c|}{ Pages download } \\
\cline { 2 - 7 } & $\mathbf{5 0 0}$ & $\mathbf{6 0 0}$ & $\mathbf{7 0 0}$ & $\mathbf{8 0 0}$ & $\mathbf{9 0 0}$ & $\mathbf{1 0 0 0}$ \\
\hline $\begin{array}{l}\text { Best- } \\
\text { First }\end{array}$ & 0.34 & 0.34 & 0.34 & 0.35 & 0.35 & 0.36 \\
\hline $\begin{array}{l}\text { Shark- } \\
\text { Search }\end{array}$ & 0.41 & 0.40 & 0.38 & 0.37 & 0.36 & 0.35 \\
\hline $\begin{array}{l}\text { LDA- } \\
\text { DBN }\end{array}$ & 0.54 & 0.51 & 0.49 & 0.49 & 0.48 & 0.46 \\
\hline
\end{tabular}

As can be seen from Table I, the target of the recall method was significantly higher than the other three algorithms, and Shark-Search convergence faster. It also can be seen from Table II, the algorithm gains significantly better than the other two algorithms. As we can be seen, this algorithm has better performance when crawling topic page.

\section{SUMMARY}

There are two core issues relating to topic crawler, link content speculation and link clustering speculation. This article focuses on the link content speculated and proposed the improved Q-learning model based on previous results and demonstrate. The experimental results show the feasibility and effectiveness of the algorithm. In the next study, Link clustering speculation need to borrow in-depth study of the theory of complex network structures.

\section{REFERENCES}

[1] C.Zhang and J.Gao,"The rough attribute graph model of Web social network and its application,"Computer Engineering and Science, vol.36, pp.517-523,Mar 2014.

[2] M.Blei,Y.Ng and I.Jordan,"Latent Dirichlet allocation,"Joumal of Machine Learning Research,vol.3, pp.993-1022,Mar 2003.

[3] F.Menczer,"Web Crawling,"in Web Data:Exploring Hyperlinks, Contents and Usage Data,2nd ed, vol.3, New York:Springer, 2011,pp.311-362.

[4] A. Pinsky and S. Karlin,"Continuous Times Markov Chains," in An Introduction to Stochastic Modeling, 4th ed, vol.6, Singapore: ElsevierInc, 2013, pp.277-346.

[5] P.Min,J.Huang,J.Zhu,J.Huang and J.Liu,"Mass of Short Texts Clustering and Topic Extraction Based on Frequent Itemsets,"Journal of Computer Research and Development,vol.52,pp.1991-1953,Sep 2015.

[6] Z.Zhang,D.Miao and C.Gao,"Short text using latent Dirichlet location,"Joumal of Computer Applicatons,vol.33,pp.1587-1590,Jun 2013.

[7] S.Hou,"Theme and deep belief network model based on the text classification method research,"MD theis,University of Yunnan, Kunming, China,2015.

[8] J.Zhai,"Computational Model of Semantic Relevancy Based on Deep Belif Net," Science Technology and Engineering,vol.14,pp.5862,Nov2014. 